\title{
TRANSPLANTATION
}

\section{PARADIGM SHIFT IN} GRAFT EVALUATION

The value of routine measurement of the intrarenal resistive index, for evaluation of renal grafts in kidney transplant recipients, has been called into question by findings recently published by researchers at the University of Leuven, Belgium. "In contrast to what has been suggested previously, we found that the intrarenal resistive index is not associated with deathcensored graft survival or graft function deterioration," comments lead investigator Maarten Naesens.

The intrarenal resistive index-a sonographic measurement used to noninvasively evaluate renal vascular resistance and elasticity—is measured in renal transplant recipients worldwide, owing to its reported correlation with graft survival and function. However, a subsequent study showed no association between this parameter and histological damage in renal allografts. Accordingly, Naesens' team conducted a large, singlecentre, prospective, observational study involving 321 renal allograft recipients to evaluate the prognostic performance of the intrarenal resistive index. The index was measured at baseline and at the time of protocol-specified graft biopsies performed in the first 2 years after transplantation.

The researchers found that the intrarenal resistive index was not associated with either acute or chronic histological damage to the allograft at the time of protocol-specified allograft biopsies. However, recipients with an intrarenal resistive index $\geq 0.8$ had higher mortality than those with an intrarenal resistive index $<0.8$, at 3 months, 12 months and 24 months post-transplantation. Interestingly, a higher intrarenal resistive index was strongly associated with older recipient age. Higher pulse pressure, lower mean blood pressure, use of $\beta$-blockers and use of diuretics were also associated with a higher intrarenal resistive index, suggesting that central haemodynamic factors have an influence.

The researchers conclude that the intrarenal resistive index reflects recipient characteristics (age and haemodynamic factors) rather than kidney damage. "Our study provides a paradigm shift in the clinical interpretation of the intrarenal resistive index after transplantation," says Naesens.

David Holmes

Original article Naesens, M. et al. Intrarenal resistive index after renal transplantation. N. Engl. J. Med. 369,

1797-1806 (2013) 